Research article

Open Access

\title{
Role of Notch signaling in cell-fate determination of human mammary stem/progenitor cells
}

\author{
Gabriela Dontu, Kyle W Jackson, Erin McNicholas, Mari J Kawamura, Wissam M Abdallah and \\ Max S Wicha
}

Comprehensive Cancer Center, Department of Internal Medicine, University of Michigan, Ann Arbor, Michigan, USA

Corresponding author: Gabriela Dontu, gdontu@umich.edu

Received: 6 Apr 2004 Revisions requested: 10 May 2004 Revisions received: 28 Jun 2004 Accepted: 15 Jul 2004 Published: 16 Aug 2004

Breast Cancer Res 2004, 6:R605-R615 (DOI 10.1186/bcr920)

(c) 2004 Dontu et al.; licensee BioMed Central Ltd. This is an Open Access article: verbatim copying and redistribution of this article are permitted in all media for any purpose, provided this notice is preserved along with the article's original URL.

\begin{abstract}
Introduction Notch signaling has been implicated in the regulation of cell-fate decisions such as self-renewal of adult stem cells and differentiation of progenitor cells along a particular lineage. Moreover, depending on the cellular and developmental context, the Notch pathway acts as a regulator of cell survival and cell proliferation. Abnormal expression of Notch receptors has been found in different types of epithelial metaplastic lesions and neoplastic lesions, suggesting that Notch may act as a proto-oncogene. The vertebrate Notch 1 and Notch4 homologs are involved in normal development of the mammary gland, and mutated forms of these genes are associated with development of mouse mammary tumors.

Methods In order to determine the role of Notch signaling in mammary cell-fate determination, we have utilized a newly described in vitro system in which mammary stem/progenitor cells can be cultured in suspension as nonadherent 'mammospheres'. Notch signaling was activated using exogenous ligands, or was inhibited using previously characterized Notch signaling antagonists.

Results Utilizing this system, we demonstrate that Notch signaling can act on mammary stem cells to promote self-

renewal and on early progenitor cells to promote their proliferation, as demonstrated by a 10-fold increase in secondary mammosphere formation upon addition of a Notchactivating DSL peptide. In addition to acting on stem cells, Notch signaling is also able to act on multipotent progenitor cells, facilitating myoepithelial lineage-specific commitment and proliferation. Stimulation of this pathway also promotes branching morphogenesis in three-dimensional Matrigel cultures. These effects are completely inhibited by a Notch4 blocking antibody or a gamma secretase inhibitor that blocks Notch processing. In contrast to the effects of Notch signaling on mammary stem/progenitor cells, modulation of this pathway has no discernable effect on fully committed, differentiated, mammary epithelial cells.

Conclusion These studies suggest that Notch signaling plays a critical role in normal human mammary development by acting on both stem cells and progenitor cells, affecting self-renewal and lineage-specific differentiation. Based on these findings we propose that abnormal Notch signaling may contribute to mammary carcinogenesis by deregulating the self-renewal of normal mammary stem cells.
\end{abstract}

Keywords: mammary gland development, mammary progenitor cells, mammary stem cells, Notch

\section{Introduction}

Stem cells in adult tissues are characterized by their ability to undergo self-renewal and multilineage differentiation [1]. The elucidation of pathways that govern stem cell functions is essential for understanding normal development and organogenesis. Moreover, there is increasing evidence that defects in these pathways play an important role in carcinogenesis [2]. The isolation of stem cells from the mammary gland in humans and rodents has been hindered by the lack of identified specific cell surface markers. Furthermore, investigation of the mechanisms underlying cell-fate decisions in mammary stem/progenitor cells has been limited by the lack of suitable in vitro culture systems, which maintain these cells in an undifferentiated state [3].

We have recently described an in vitro culture system that allows for the propagation of primary human mammary epithelial stem cells and progenitor cells in an undifferentiated 
state, based on their ability to proliferate in suspension as spherical structures, which we have termed 'nonadherent mammospheres' [4]. As has previously been described for neuronal stem cells and progenitor cells cultured as neurospheres [5], we have demonstrated that mammospheres are composed of stem cells and progenitor cells capable of self-renewal and multilineage differentiation [4]. We have utilized this system to investigate the role of Notch signaling in mammary cell-fate determination.

In other systems, Notch signaling has been shown to play an important role in cell-fate determination, as well as in cell survival and proliferation $[6,7]$. The Notch proteins, represented by four homologs in mammals (Notch1-Notch4), interact with a number of surface-bound or secreted ligands (Delta-like 1, Delta-like 3, Delta-like 4, Jagged 1 and Jagged 2) [8-10]. These interactions are modulated by modifier proteins from the Fringe family (Lunatic, Manic, and Radical Fringe) [11]. Upon ligand binding, Notch receptors are activated by serial cleavage events involving members of the ADAM protease family, as well as an intramembranous cleavage regulated by gamma secretase (presinilin). This intramembranous cleavage is followed by translocation of the intracellular domain on Notch to the nucleus, where it acts on downstream targets [11]. The vertebrate Notch4 gene has been shown to be involved in normal mammary development [12]. In vitro, overexpression of a constitutively active form of Notch4 inhibits differentiation of normal breast epithelial cells [13]. In vivo, transgenic mice expressing a constitutively active form of Notch4 fail to develop normal mammary glands and subsequently develop mammary tumors [14]. These studies suggest the involvement of Notch signaling in normal breast development, and that alterations in Notch signaling may play a role in breast cancer development $[15,16]$.

\section{Materials and methods Dissociation of mammary tissue}

Normal breast tissue from reduction mammoplasties was dissociated mechanically and enzymatically, as previously described [17]. Cells were sieved sequentially through a $100 \mu \mathrm{m}$ cell strainer and a $40 \mu \mathrm{m}$ cell strainer (BD Falcon, Palo Alto, CA, USA) to obtain a single cell suspension.

\section{Mammosphere culture}

Single cells were plated on ultralow attachment plates (Corning, Acton, MA, USA) at a density of 20,000 viable cells $/ \mathrm{ml}$ in primary culture and a density of $1000 \mathrm{cell} / \mathrm{s} / \mathrm{ml}$ in passages. Cells were grown in serum-free mammary epithelial growth medium (BioWhittaker, Rockland, ME, USA), supplemented with B27 (Invitrogen, Carlsbad, CA, USA) and $10 \mathrm{ng} / \mathrm{ml}$ epidermal growth factor (BD Biosciences, Palo Alto, CA, USA). The bovine pituitary extract was excluded.
Mammospheres were collected by gentle centrifugation (800 rpm) after 7-10 days, and were dissociated enzymatically (10 min in $0.05 \%$ trypsin, $0.53 \mathrm{mM}$ EDTA-4Na; Invitrogen) and mechanically, using a fire-polished Pasteur pipette. The cells obtained from dissociation were sieved through a $40 \mu \mathrm{m}$ sieve and analyzed microscopically for single cellularity. Cells plated at low densities (1000 cells $/ \mathrm{ml}$ ) were grown in conditioned medium from high-density primary cultures in suspension.

\section{Differentiating culture conditions}

Single cell suspensions were plated on collagen-coated plates at a density of 2000 viable cells $/ 10 \mathrm{~cm}$ diameter dish. Cells were grown in Ham's F-12 medium (BioWhittaker) with $5 \%$ fetal bovine serum (FBS), $5 \mu \mathrm{g} / \mathrm{ml}$ insulin, 1 $\mu \mathrm{g} / \mathrm{ml}$ hydrocortisone, $10 \mu \mathrm{g} / \mathrm{ml}$ cholera toxin (Sigma, St Louis, MO, USA), $10 \mathrm{ng} / \mathrm{ml}$ epidermal growth factor (BD Biosciences) and $1 \times$ Pen/Strep/Fungizone Mix (BioWhittaker). Three-dimensional cultures in Matrigel were established as previously described [18].

\section{Immunostaining}

To assess the lineage composition of the colonies, cells were fixed on plates for $20 \mathrm{~min}$ in methanol, at $-20^{\circ} \mathrm{C}$, and were then stained using Peroxidase Histostain-Plus and Alkaline-phosphatase Histostain-Plus kits (Zymed, South San Francisco, CA, USA), according to the manufacturer's protocol. The ductal-alveolar structures were fixed in Matrigel in $4 \%$ neutral formalin, and treated with $1 \mathrm{mg} / \mathrm{ml}$ proteinase $\mathrm{K}$ for 10-15 min at room temperature, prior to staining. For immunostaining in Matrigel, all antibody incubations were for $60-90 \mathrm{~min}$ at $37^{\circ} \mathrm{C}$, and washes were for $20 \mathrm{~min}$ at $37^{\circ} \mathrm{C}$, with a final overnight wash at $4^{\circ} \mathrm{C}$.

The primary antibodies - epithelial-specific antigen and cytokeratin 18 for epithelial cells, CD10 and cytokeratin 14 (Novocastra, Newcastle-upon-Tyne, UK) for myoepithelial cells - were used at the dilutions indicated by the manufacturer. The Notch4 antibody was used at a dilution of 1:50 for immunohistochemistry and of 1:20 for immunostaining structures within Matrigel. AEC and DAB (Zymed) were used as substrates for peroxidase, and NBT/BCIP (Gibco/ BRL, Gaithersburg, MD, USA) was used for alkaline phosphatase. FITC-labeled and Texas-red labeled secondary antibodies (Jackson Labs, West Grove, PA, USA) were used for fluorescent microscopy.

\section{Luciferase gene reporter assay}

MCF-7 cells were grown in RPMI 10\% FBS on tissue culture-treated plastic dishes. Cells were collected by trypsinization and RNA was isolated as previously described and used to test expression of Notch1 and Notch4 by RT-PCR. MCF-7 cells expressed both Notch1 and Notch4 so they were further used for the gene reporter assay. Expression of Notch4 in MCF-7 cells was also confirmed by 
immunohistochemistry. The staining was mostly nuclear and cytoplasmic, although membrane staining was also present, confirming detectable levels of active Notch4 in MCF-7 cells.

The specificity of the antibody was tested by the manufacturer via western blotting using cell lysates from a variety of human cancer cell lines (Jurkat cells, HeLa, H4, etc.) that express all four homologs of Notch. The antibody was raised against a synthetic peptide that does not show sequence similarity with any of the other Notch members, as shown by Blast search analysis. Using the Lipofectamine-Plus kit (Promega, Madison, WI, USA), cells were co-transfected with pcDNA3 (Invitrogen) and a pGL3-promoter vector (Promega) containing a Hes-1 promoter-derived sequence upstream of luciferase, according to the manufacturer's protocol. Stable transfection was achieved by selecting with $800 \mu \mathrm{g} / \mathrm{ml}$ Geneticin (Gibco/ BRL).

Cells were incubated for 4,8 and 16 hours in the presence of agonists or antagonists of Notch signaling as follows: blocking Notch4 antibody (Santa-Cruz, Santa Cruz, CA, USA) was used in dilutions of 1:100, 1:50, 1:25 and 1:10, gamma secretase inhibitor (GSI) type I (Z-Leu-Leu-Nle$\mathrm{CHO}$; Calbiochem, San Diego, CA, USA) was used at 20 $\mu \mathrm{M}, 10 \mu \mathrm{M}, 5 \mu \mathrm{M}, 1 \mu \mathrm{M}, 100 \mathrm{nM}$ and $10 \mathrm{nM}$ concentration, and the DSL peptide (CDDYYYGFGCNKFCRPR; Research Genetics, Huntsville, AL, USA) was used at 10 $\mu \mathrm{M}, 1 \mu \mathrm{M}, 100 \mathrm{nM}, 10 \mathrm{nM}$ and $1 \mathrm{nM}$. The control scrambled peptide (DYGKRCYGCFPDYFNCR) was used at 10 $\mu \mathrm{M}$ concentration. Dimethylsulfoxide 1:50 was used as a control for GSI. Direct evidence that GSI blocks Notch4 and Notch1 cleavage in vitro has been provided previously [19].

The treatment with antagonist involved incubating for 1 hour at $37^{\circ} \mathrm{C}$, prior to treatment with the DSL peptide. Incubation of the antibody with the peptide against which it was raised (Santa Cruz) was carried out at antibody to peptide ratios of 1:1,1:2.1:3 and 1:4 (vol:vol). Luciferase activity was assessed using the Luciferase assay system (Promega) and a Turner Designs luminometer TD-20/20 (Turner Designs, Sunnyvale, CA, USA).

\section{Treatment with Notch agonists and antagonists}

Treatment with Notch agonists and antagonists was carried out as described for the reporter assay. The DSL peptide was used at a concentration of $100 \mathrm{nM}$. The recombinant Delta $1 \mathrm{Fc}$ ligand was prepared and used as previously described [20]. Anti-human Fc antibody (Jackson Labs) was added to the medium at 1:100 dilution, to cluster the recombinant ligand-receptor complexes. The blocking Notch4 antibody was used at a dilution of 1:50, and the GSI at a concentration of $5 \mu \mathrm{M}$. In experiments in which treatment was applied for longer than 48 hours, it was added every 2 or 3 days when the medium was changed. The Notch4 antibody and antigenic peptide used for treating cells in culture did not contain sodium azide.

\section{FACS analysis}

Cells were fixed in methanol and immunostained with antiepithelial-specific antigen, CD10 and Muc1 antibodies (Novocastra), at the concentration recommended by the manufacturer. Incubation was performed for $30 \mathrm{~min}$ at room temperature in Hanks Balanced Salt Solution (HBSS) with $2 \%$ FBS, followed by washing in HBSS with $2 \%$ FBS. The same procedure was followed for staining with secondary antibody, anti-goat $\lg G$ or anti-rabbit lgG, labeled with FITC (1:200 dilution; Jackson Labs). After incubation, cells were washed once with HBSS (BioWhittaker) and were resuspended in HBSS supplemented with 5\% FBS. Cells were stained with $1 \mu \mathrm{g} / \mathrm{ml}$ propidium iodide (Sigma) for $30 \mathrm{~min}$ for DNA cell cycle analysis or for $5 \mathrm{~min}$ for viability. Analysis was performed using FACStarPLUS (Becton Dickinson, Palo Alto, CA, USA) and the Elite SP (Becton Dickinson) flow cytometer.

\section{Results \\ Experimental strategy}

In order to investigate the role of the Notch pathway in the development of the mammary gland, we examined the effect of agonists and antagonists of Notch signaling on cell-fate determination utilizing mammary stem/progenitor cells cultured in suspension, as mammospheres, and differentiated mammary cells, obtained by passaging these cells on a collagen substratum. The experimental strategy is outlined in Fig. 1. The activators and inhibitors of Notch signaling were applied at three different stages of in vitro development, which we have previously characterized [4]. First, the treatment was applied in suspension culture, which is enriched in stem/progenitor cells (self-renewal of stem cells and the proliferation of progenitor cells occur in these conditions) (Fig. 1a). Second, the treatment was applied in cultures on collagen substratum, under conditions that promote differentiation of mammosphere-derived cells (multipotent progenitors commit to a specific lineage) (Fig. 1b). Finally, the treatment was applied in cultures on a collagen substratum, using differentiated cells passaged on a collagen substratum (lineage-restricted progenitors undergo terminal differentiation) (Fig. 1c).

A synthetic peptide derived from the DSL (Delta-SerrateLAG 2) domain conserved in all Notch ligands [9] and a previously described recombinant Delta 1 ligand fused to the immunoglobulin $\mathrm{Fc}$ fragment (used in combination with an anti-human Fc antibody, for ligand clustering) [20] were utilized as agonists of Notch signaling. A Notch4-specific antibody or a GSI that blocks the intramembranous cleavage of Notch, required for signaling [21], was used as the 
Figure 1

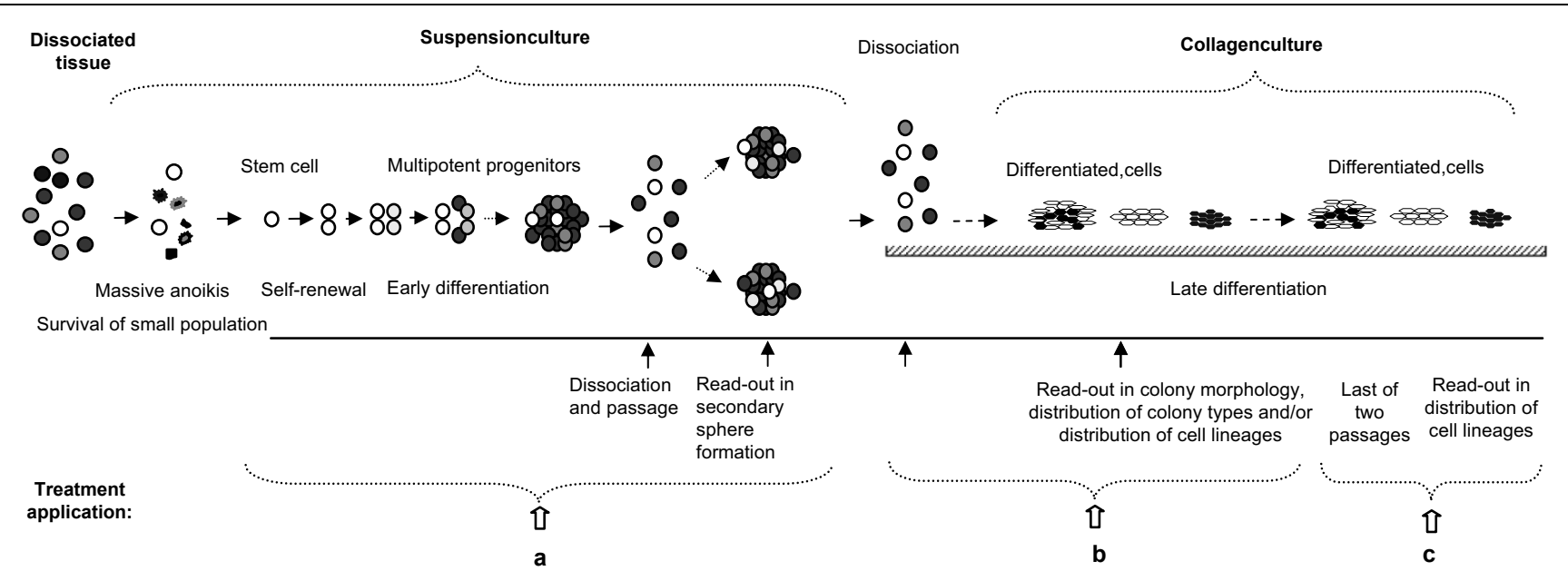

Effect of modulators of Notch signaling on self-renewal of mammary stem cells and on lineage commitment of mammary progenitor cells: experimental strategy. Treatment was applied (a) to mammospheres in suspension culture, (b) to mammosphere-derived cells cultivated on a collagen substratum, and (c) to differentiated cells cultivated on a collagen substratum. The assay for self-renewal was mammosphere formation. The assay for multilineage potential or lineage commitment was lineage-specific immunostaining of clonogenic cultures on the collagen substratum.

antagonist. The synthetic DSL peptide, the recombinant Delta fused to Fc and GSI are all well characterized with respect to their effect on Notch signaling $[9,20]$. We utilized a reporter assay in MCF-7 cells, in which luciferase expression was driven by the Hes- 1 promotor, a gene regulated by Notch signaling [22], to confirm the ability of activating or inhibiting ligands to affect Notch signaling.

As shown in Fig. 2a, DSL peptide upregulated Hes-1driven luciferase expression. These results are consistent with previous characterization of these Notch signaling agonists in reporter assays and in experiments using other cell types [9]. Notch signaling was blocked using a Notch4-specific antibody, or a GSI, which abrogates the intramembranous cleavage of Notch, required for signaling $[19,21]$. Both of these inhibitors blocked endogenous Hes1-driven luciferase expression, as well as the increase in luciferase expression induced by the DSL ligand (Fig. 2a). The specificity of the blocking antibody was confirmed by successful competition with the antigenic peptide used to produce the antibody (data not shown). Furthermore, these effects were dose dependent (Fig. 2b).

\section{Effects of Notch signaling on self-renewal of mammary stem cells}

We examined the effects of these agonists and antagonists of Notch signaling on primary, secondary and tertiary mammosphere formation (Fig. 1a). This assay has previously been used for the in vitro study of neural stem cell selfrenewal [19] and relies on the observation that neurosphere formation is initiated clonally by neural stem cells [5].
Utilizing retroviral marking studies, we also have demonstrated that mammospheres are clonally derived and do not result from cellular aggregation [4]. Primary, secondary, and tertiary generation mammospheres were formed in the presence or absence of the Notch-activating DSL peptide. Although DSL peptide had only modest effects on primary mammosphere formation, it increased secondary and tertiary mammosphere formation 10-fold, compared with control cultures (Fig. 3a,3b,3d,3e,3g). If this effect was due to stem cell self-renewal, we would predict that cells derived from these spheres would retain their multipotent differentiation ability. We therefore examined the ability of secondary and tertiary mammosphere-derived cells to clonally differentiate along the multiple lineages present in the adult mammary gland.

The adult mammary gland has a lobuloalveolar structure composed of three cell lineages: myoepithelial cells that form the basal layer of ducts and alveoli, ductal epithelial cells that line the lumen of ducts, and alveolar epithelial cells that synthesize milk proteins [23]. Mammospheres grown in the presence or absence of Notch agonists or antagonists were dissociated into single cells and plated at clonogenic densities on collagen substrata in a medium that promotes differentiation [4]. After 7 days of cultivation, the clonally derived colonies were immunostained using lineage-specific markers (epithelial-specific antigen and cytokeratin 18 for ductal epithelial cells, and cytokeratin 14 and smooth muscle actin for myoepithelial cells). The number of multipotent cells after one passage was seven times higher, and that after two passages 100 times higher, in mammospheres cultured in the presence of DSL, 

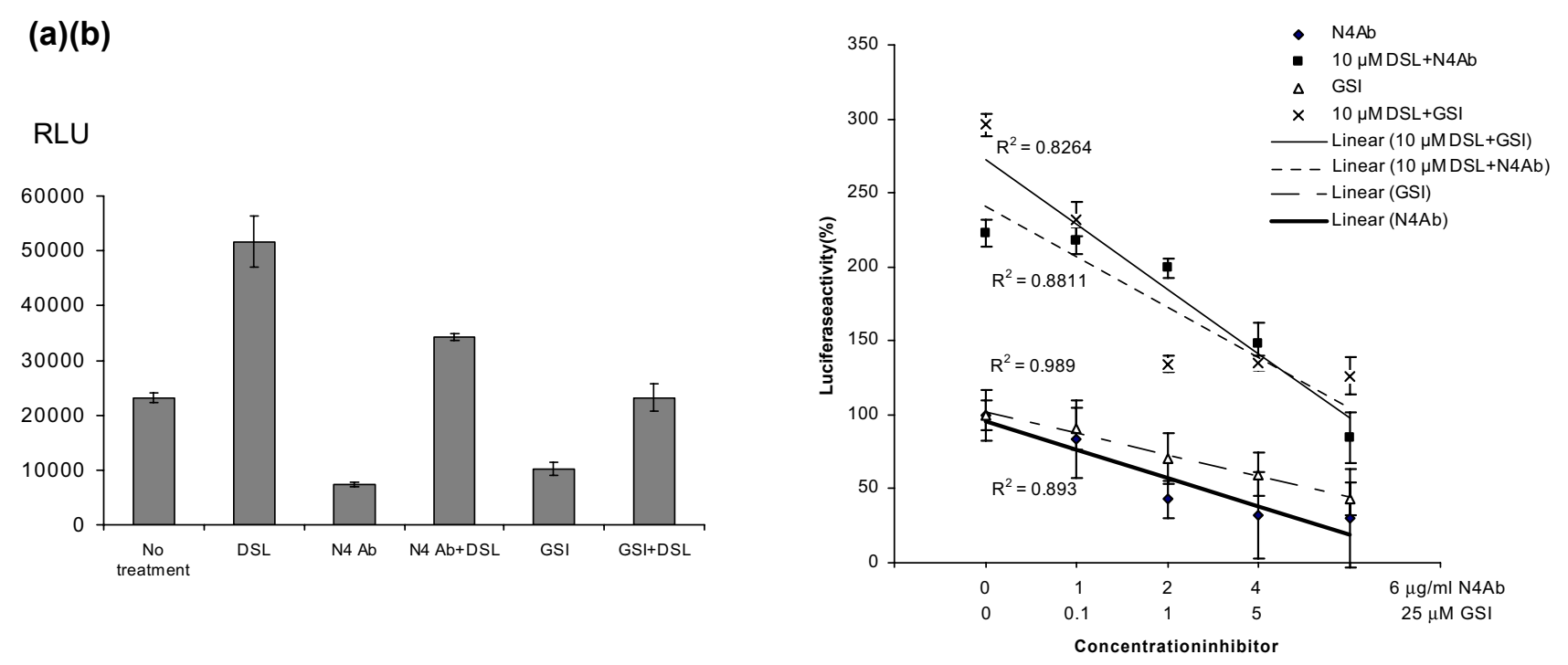

Effect of Notch agonist and antagonist treatment, assessed by Hes-1-driven luciferase expression in MCF-7 cells. (a) DSL treatment (100 nM) increased luciferase expression twofold compared with control, whereas treatment with the N4 blocking antibody (N4Ab) or gamma secretase inhibitor (GSI) $(5 \mu \mathrm{M})$ reduced both endogenous and DSL-induced luciferase expression. (b) Dose-effect of Notch signaling antagonists. The difference in luciferase activity in cells treated with the maximum dose of inhibitory agent and in nontreated cells cells was statistically significant for each of the four different treatments $(P<0.005)$. The $R^{2}$ values and trendlines for the four sets of experiments are shown.

compared with mammospheres cultured without added DSL. These calculations are based on the number of spheres (Fig. $3 \mathrm{~g}$ ) and the corresponding percentage of multilineage colonies, in each experiment (Fig. 3h).

The ability of Notch activating ligands to increase mammosphere formation, as well as the production of multilineage progenitors, suggests that Notch activation promotes mammary stem cell self-renewal. Similar effects to the DSL peptide on mammosphere formation were obtained with recombinant Delta Fc fragment (data not shown). In addition to increasing the number of mammosphere initiating cells and bipotent progenitor cells, treatment with Notch agonists increased their proliferation potential, as shown by the increased size of mixed lineage colonies, generated clonally on collagen culture by these cells (Fig. 3i). Treatment with Notch agonists was applied in suspension culture only, and was not applied to sphere-derived cells subsequently cultured on collagen substrata, suggesting that the effect on proliferation is irreversible. This positive effect of Notch activation on proliferation potential of stem/ progenitor cells also occurs in the mammospheres, as demonstrated by an increased size of the primary and secondary mammosphere diameter in the DSL-treated suspension cultures (mean \pm standard error of the mean, $245 \pm$ $11.5 \mu \mathrm{M}$ versus $130 \pm 3 \mu \mathrm{M} ; n=69$ and $n=89)$. This is due to an increased number of cells per sphere, as demonstrated by cell counts that are approximately double in DSL-treated mammospheres compared with control cul- tures. The viability of cells within a mammosphere was similar in the presence of and the absence of DSL. These results indicate that the Notch activation increases proliferation of progenitor cells.

In order to further investigate the role of Notch signaling, and in particular Notch4, in mammary stem cell selfrenewal, we assayed the effect of Notch inhibitors on mammosphere formation. The addition of a Notch4 blocking antibody to primary cultures had no effect on primary sphere formation, but completely abolished secondary mammosphere formation (Fig. 3a,3c,3d,3f,3h). A similar effect was obtained by blocking Notch activation with a GSI at 1-5 $\mu \mathrm{M}$, a concentration found to effectively block Notch activation in the luciferase reporter assay (Fig. 2a). In contrast to the effect of adding Notch4 blocking antibody at the time of mammosphere formation, this treatment had no effect when added 24-48 hours after mammosphere formation. This suggests that the effects of Notch activation on mammary stem cell self-renewal may occur during the initial stages of mammosphere formation. The cells responding to modulation of Notch signaling are the sphere initiating cells, capable of survival and proliferation in suspension.

\section{Effect of Notch signaling on lineage specification of mammary progenitor cells}

In other developing systems, Notch signaling has been shown to effect lineage commitment as well as stem cell 
Figure 3

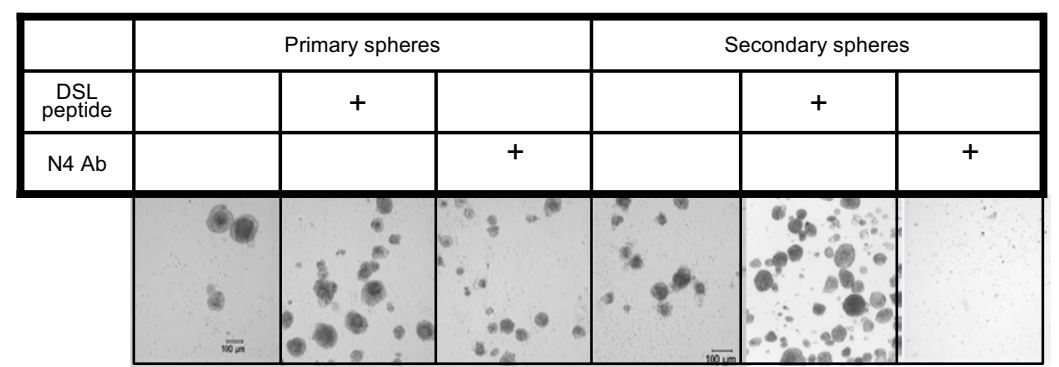

$(a)(b)(c)(d)(e)(f)$

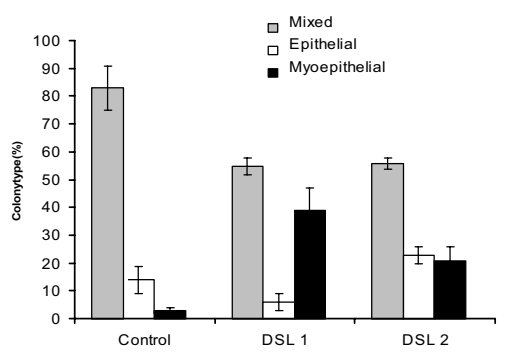

(h)

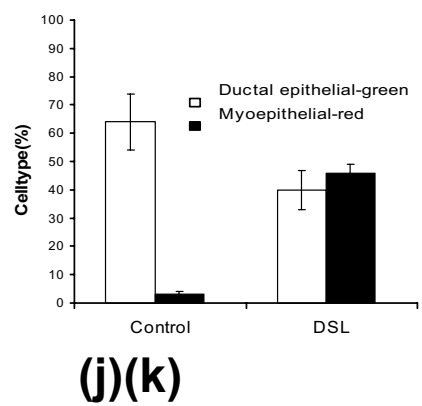

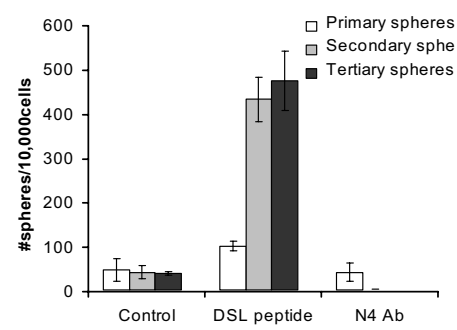

(g)

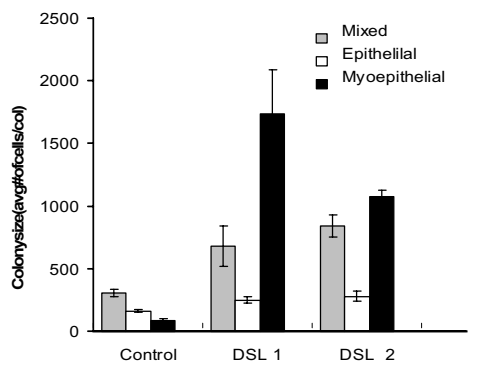

(i)

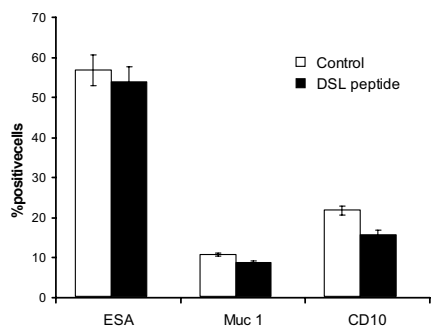

Effect of modulators of Notch signaling on self-renewal of mammary stem cells and on lineage commitment of mammary progenitor cells. (a)-(g) Effect of Notch agonist and antagonist treatment on primary and secondary mammosphere formation. Primary and secondary mammospheres were grown in standard conditions $(a, d)$, in the presence of DSL peptide $(b, e)$ and in the presence of N4 blocking antibody (N4Ab) (c, f). Primary mammospheres grown in the presence of N4 antibody (c) are smaller than the control (a). Secondary mammosphere formation is completely blocked by treatment with Notch4 blocking antibody $(\mathrm{d}, \mathrm{f})$. The addition of DSL peptide increases primary, secondary and tertiary mammosphere formation $(a, b$, $d, e, g$ ). Data are presented as the mean \pm standard error of the mean. The calculated number of multilineage progenitor cells (number of spheres $x$ $\%$ multilineage progenitors) shows a sevenfold increase after one passage, and a 100-fold increase after two passages, in mammospheres cultured in the presence of DSL compared with mammospheres cultured without added DSL. (h)-(k) Effect of Notch activation on lineage specification of human mammary progenitor cells. DSL treatment of mammospheres in suspension culture (DSL1) and on mammosphere-derived cells on the collagen substratum (DSL2) increases the number of myoepithelial progenitors, as shown by the clonogenic assay (h), and increases the rate of proliferation of bipotent and myoepithelial progenitors, as reflected by the colony size (i). DSL treatment in suspension culture increases the percentage of myoepithelial cells, as shown by flow-cytometry analysis (j). Cells were stained red (phycoerythrin [PE]) with myoepithelial marker CD10, and green (FITC) with the ductal epithelial marker epithelial-specific antigen (ESA). Treatment with DSL peptide does not have a lineage selective effect on differentiated cells, as shown by flow-cytometry analysis of human mammary epithelial cells passaged twice on collagen ( $k$ ). Data are presented as the mean \pm standard error of the mean.

self-renewal $[20,24-26]$. The inability of previously described culture systems of mammary cells to maintain progenitor cells in an uncommitted state has limited the in vitro study of lineage commitment. Since we have previously demonstrated that, in addition to mammary stem cells, mammospheres contain mammary progenitors capa- ble of multilineage differentiation, we utilized this system to determine whether Notch signaling could also act on mammary progenitor cells to affect lineage-specific commitment. In order to test this, we added the DSL peptide to mammosphere cultures (Fig. 1a), to cultures of mammosphere-derived cells on collagen substrata in conditions that 
promote their differentiation (Fig. 1b) or to cultures of differentiated cells (Fig. 1c).

Cells were assayed for lineage-specific commitment, using the clonogenic assay described earlier. As shown in Fig. 3h (DSL1), the percentage of myoepithelial progenitor cells generated by DSL-treated mammospheres was increased approximately 13-fold compared with cells from mammospheres cultured in the absence of DSL ligand. The percentage of myoepithelial progenitor cells generated by mammosphere-derived cells treated with DSL starting from the moment of their plating on collagen culture (DSL2) generated sevenfold more myoepithelial progenitor cells compared with controls. The significant effect of DSL peptide, when added only to suspension-cultured mammospheres, suggests that Notch activation increased the number of myoepithelial progenitors and/or their rate of proliferation in an irreversible manner. The increased size of myoepithelial and bilineage colonies (Fig. 3i) formed in the presence of DSL peptide suggests that Notch stimulation may promote proliferation of myoepithelial and bilineage progenitors.

In order to determine whether the increase in colony size results from an effect of Notch on cell survival, we used propidium iodide staining in the same clonogenic assay. Treatment with DSL peptide did not have a discernable effect on the cell-death rate of either ductal epithelial cells or myoepithelial cells. To assess more accurately the increase in total number of myoepithelial cells upon treatment with DSL in suspension culture, we repeated the same experiment described earlier, utilizing flow cytometry analysis, to quantitate the cell lineage (Fig. 3j). The results showed a 15-fold increase in myoepithelial cells upon addition of DSL peptide to the culture.

\section{Effect of Notch signaling on differentiated mammary epithelial cells}

In contrast to the significant effects of Notch signaling on uncommitted mammary cells, modulation of the Notch pathway had no significant effect on differentiated mammary epithelial cells. The addition of DSL peptide to differentiated cells cultured on collagen substrata (Fig. 1c) had no effect on lineage specification, as shown by flow-cytometry analysis using lineage-specific markers (Fig. 3k). Furthermore, the addition of Notch antibody or the GSI at 1$10 \mu \mathrm{M}$ had no significant effect on cell number or lineage commitment (data not shown). This also demonstrates that Notch activation is not necessary for the survival of fully differentiated mammary cells.

\section{Effect of Notch signaling on branching morphogenesis}

It has previously been demonstrated that culture of human or rodent mammary cells in reconstituted basement membrane (Matrigel) promotes morphogenic differentiation $[18,27]$. Since mammospheres are composed of undiffer- entiated mammary cells, we have utilized three-dimensional Matrigel cultures to explore the morphogenic differentiation potential of these cells. Following 3-4 weeks of cultivation in Matrigel, mammospheres develop extensive ductal lobuloalveolar structures similar in morphology to those found in vivo (Fig. 4a,4b). We utilized this system to examine the role of Notch signaling in morphogenesis. Secondary mammospheres were imbedded in Matrigel and treated with DSL peptide, Notch4 antibody or GSI. Treatment with DSL peptide promoted earlier development, as well as increased length and number of branching structures, compared with control cultures (Fig. 4c,4d). In contrast, the Notch4 antibody completely inhibited branching morphogenesis (Fig. 4c,4d). Similar effects were produced by blocking Notch signaling with GSI (data not shown).

\section{Expression of Notch4 in human mammary epithelial cells in vitro}

We and other workers have found that expression of Notch receptors in the adult human mammary gland was below the level of detection using immunostaining of tissue sections, but was detectable by RT-PCR [28]. Moreover, expression of Notch4 in dissociated mammary tissue was below the level of detection using flow cytometry. The experiments already described, however, suggested that Notch receptors are present in mammary stem cells and progenitor cells, and are downregulated in differentiated cells. Previous gene expression profile analysis by microarray and real-time RT-PCR showed that mammospherederived cells passaged in suspension culture express all Notch receptors at a higher level (twofold to fourfold) than the same cells cultured under conditions that induce differentiation [4].

These results suggest that Notch receptors are expressed at higher levels in more primitive mammary cells, such as those found in the developing mammary gland, compared with the mature gland. To test this hypothesis, we immunostained structures generated by mammospheres in Matrigel culture, at different stages of growth (between 4 days and 2 weeks). We found that cells expressing Notch4 were uniformly distributed in spherical structures at the beginning of cultivation (Fig. 5a,5b). As branching morphogenesis occurred, Notch4 expression became limited to the subterminal end of branches (Fig. 5c,5d). The corresponding areas in vivo have previously been postulated to contain mammary stem cells and progenitor cells [23].

\section{Discussion}

In order to study the role of Notch signaling in mammary cell-fate determination, we have utilized an experimental system that allows the analysis of cell self-renewal and differentiation in vitro. This enabled us to study the effect of Notch activation and inhibition at a single cell level and under conditions where the timing and the duration of 

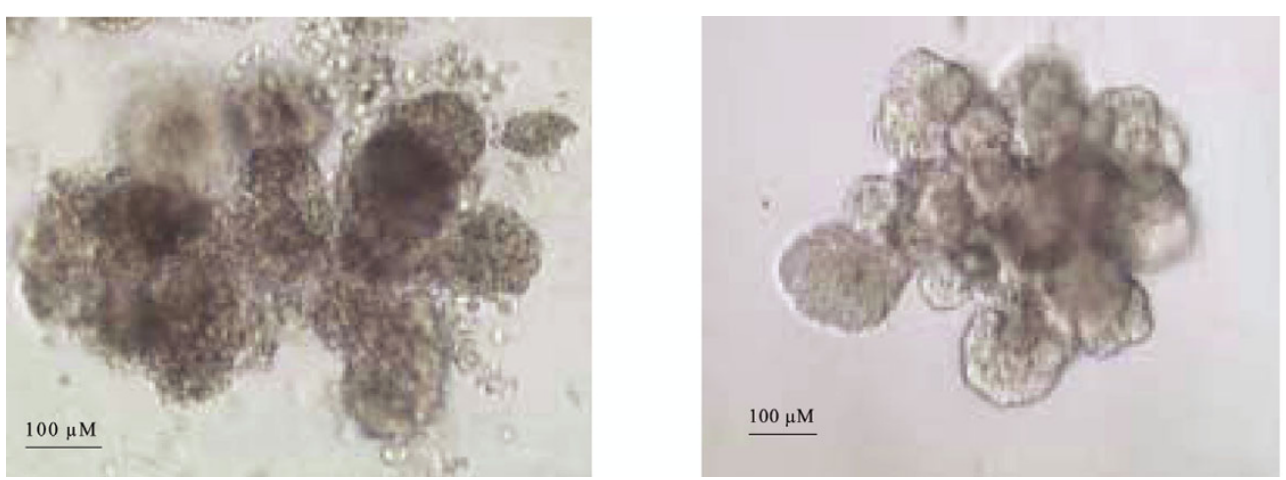

(a)(b)
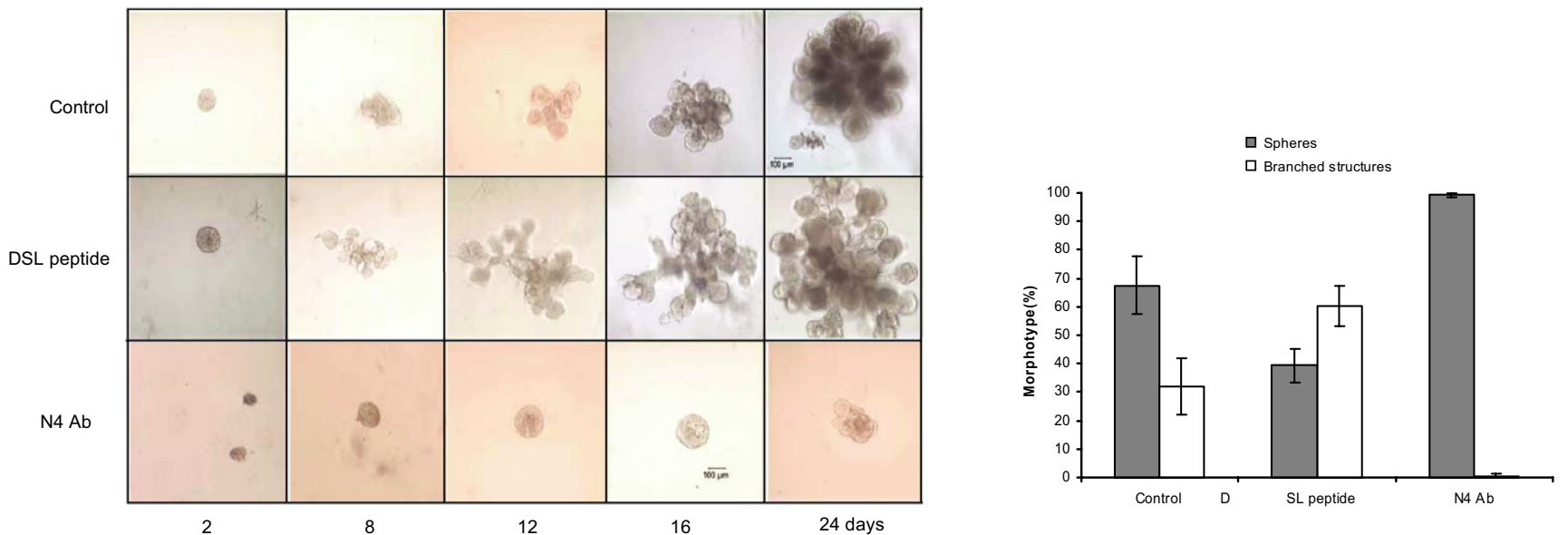

(c)(d)

Effect of Notch signaling on branching morphogenesis. (a) Fragment of the human mammary gland, after 8 hours of collagenase digestion of the reduction mammoplasty sample. (b) Complex ductal-alveolar structure developed in 18 days, from a mammosphere embedded in Matrigel. (c) Secondary mammospheres were embedded in Matrigel and treated with DSL peptide or Notch4 blocking antibody (N4Ab). Longer branches developed earlier in the presence of DSL peptide. (d) In addition, more spheres developed branching structures. The Notch4 blocking antibody completely inhibited proliferation and branching in Matrigel (c, d). Images shown in (c) are representative of 100-150 structures per experiment, scored in three different experiments with cells derived from three different patients. Data are presented as the mean \pm standard error of the mean.

Notch signaling could be controlled. Our findings suggest that Notch signaling regulates cell-fate decisions in the mammary gland at several distinct developmental stages.

We propose that in the mammary gland, as has been reported in other developmental systems, Notch activation acts as a regulator of asymmetric cell-fate decisions. Notch activation promotes self-renewal in stem cells, whereas in later stages of development it biases cell-fate decisions in mammary progenitor cells towards adoption of a myoepithelial cell fate versus an epithelial cell fate.

The increase in the number of mammosphere-initiating cells reflects an increase in the number of cells capable of selfrenewal, in the experimental system used in this study. The effect of Notch activation on mammosphere formation underscores the value of the mammosphere cultivation system in studying in vitro self-renewal regulation. It has previously been shown in suspension culture that neural stem cells can generate mixed colonies, termed neurospheres, composed of stem cells and progenitor cells. We have demonstrated that mammospheres are composed of stem cells and progenitor cells capable of self-renewal and multilineage differentiation [4]. Our clonal analysis of individual cells from single spheres suggested that mammospheres are composed of approximately 150-200 progenitor cells, which cannot generate new spheres but have bilineage or trilineage differentiation potential, and of one or two putative stem cells able to generate a new sphere with the same cellular composition as the parental sphere. 
Figure 5

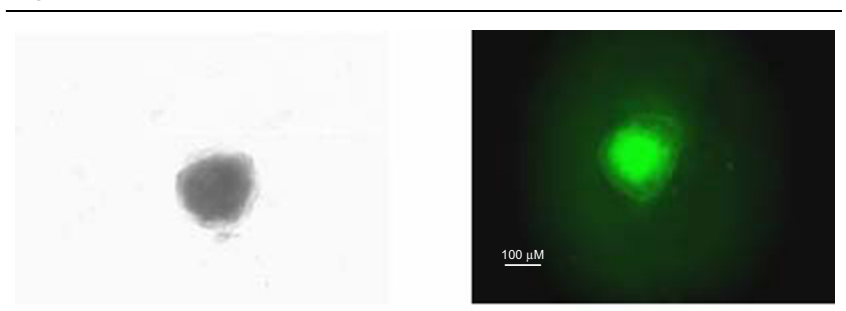

(a)(b)
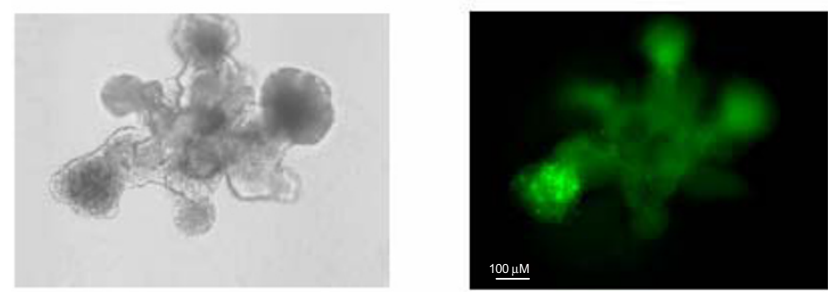

(c)(d)

Notch4 protein detected by immunostaining (FITC-green) in structures derived from mammospheres embedded in Matrigel. (a),(b) Spherical structures at 5 days of cultivation (Nomarski contrast-phase image and immunofluorescence). (c),(d) Branching structures at 16 days of cultivation.

The constant frequency of sphere formation during multiple passages, observed in passages of both individual and pooled mammospheres, suggests that only asymmetric self-divisions or a small number of symmetric self-renewal divisions contribute to sphere formation (Fig. 6a). If bipotent or monopotent progenitor cells were able to survive in suspension, they would generate mammospheres of increasingly smaller size and, eventually, they would stop growing in suspension after several passages (Fig. 6b), which did not occur in the experimental setting used here. An increase in the number of symmetric self-renewal divisions would result in an amplification of the cellular population, by progressively increasing the number of mammospheres at each passage (Fig. 6c). This effect was obtained upon exogenous activation of Notch signaling. In this discussion we adopted the operational definition of stem cells as the only normal cells capable of self-renewal $[29,30]$.

Treatment with Notch agonists increased the commitment of bilineage progenitors to myoepithelial lineage, when applied early in suspension culture (Fig. 1, DSL1) or later on collagen culture (Fig. 1, DSL2). The effect on lineage commitment appears to be irreversible, since early activation of Notch, in suspension culture, resulted in an increase in the number of myoepithelial progenitors - although the treatment with Notch agonists was not maintained on collagen cultures. In addition to an increase in the number of bipotent and myoepithelial progenitor cells, treatment with Notch agonists resulted in a significant increase in the size of both mixed and myoepithelial colonies. This sug- gests that Notch activation increases the proliferation potential of both bipotent and myoepithelial progenitors. These effects are irreversible and may involve an instructive mechanism or the selective proliferation of myoepithelial cells. In contrast to the effects of Notch signaling on mammary stem cells and progenitor cells, activation or inhibition of these pathways in fully differentiated mammary cells had no apparent effect.

The increased branching we observed in the three-dimensional cultivation system upon Notch activation is consistent with previous studies demonstrating that proliferation of myoepithelial cells can facilitate branching morphogenesis. Moreover, Notch signaling appears to be absolutely required for branching and growth in three-dimensional cultures as development of branching structures is completely blocked by Notch4 antibody or GSI. The expression of Notch4 protein in the growing mammary tree in vitro is also consistent with an important role of Notch signaling in morphogenesis of the mammary gland. The apparent discrepancy between our results and the previous findings by Uyttendaele and colleagues [13] (who showed that overexpressing Notch4 in the mouse mammary epithelial TAC-2 cell line prevented branching morphogenesis in response to hepatocyte growth factor or transforming growth factor beta) underscores the importance of the experimental systems utilized. This discrepancy may be accounted for by differences in the experimental systems, such as the use of an immortalized mouse epithelial cell line in the Uyttendaele study compared with primary normal human cells in our study. Furthermore, Uyttendaele and colleagues utilized overexpression of activated Notch, whereas we used exogenous modulation of the Notch pathway.

Smith and colleagues [12] demonstrated that Notch4 has an important role in both normal mammary development and carcinogenesis. Transgenic mice harboring a constitutively active Notch4 under mouse mammary tumor virus promoter regulation exhibited arrested mammary gland development, and eventually developed poorly differentiated adenocarcinomas. Questions remain in interpreting the report that the Notch4 knockout mouse has no apparent defect in mammary gland development [31]. This may indicate that in vivo there are compensatory mechanisms absent in our in vitro experimental system. Alternatively, the expression or activity of different homologs of Notch can be interdependent, as suggested by previous studies [32].

\section{Conclusions}

Our findings regarding the role of Notch in promoting selfrenewal of mammary stem cells, in addition to previous observations that it can function as a proto-oncogene [15,16,28,33-38], suggest that abnormal Notch signaling may be involved in carcinogenesis, through deregulation of normal mammary stem cell self-renewal. Indeed, it has 


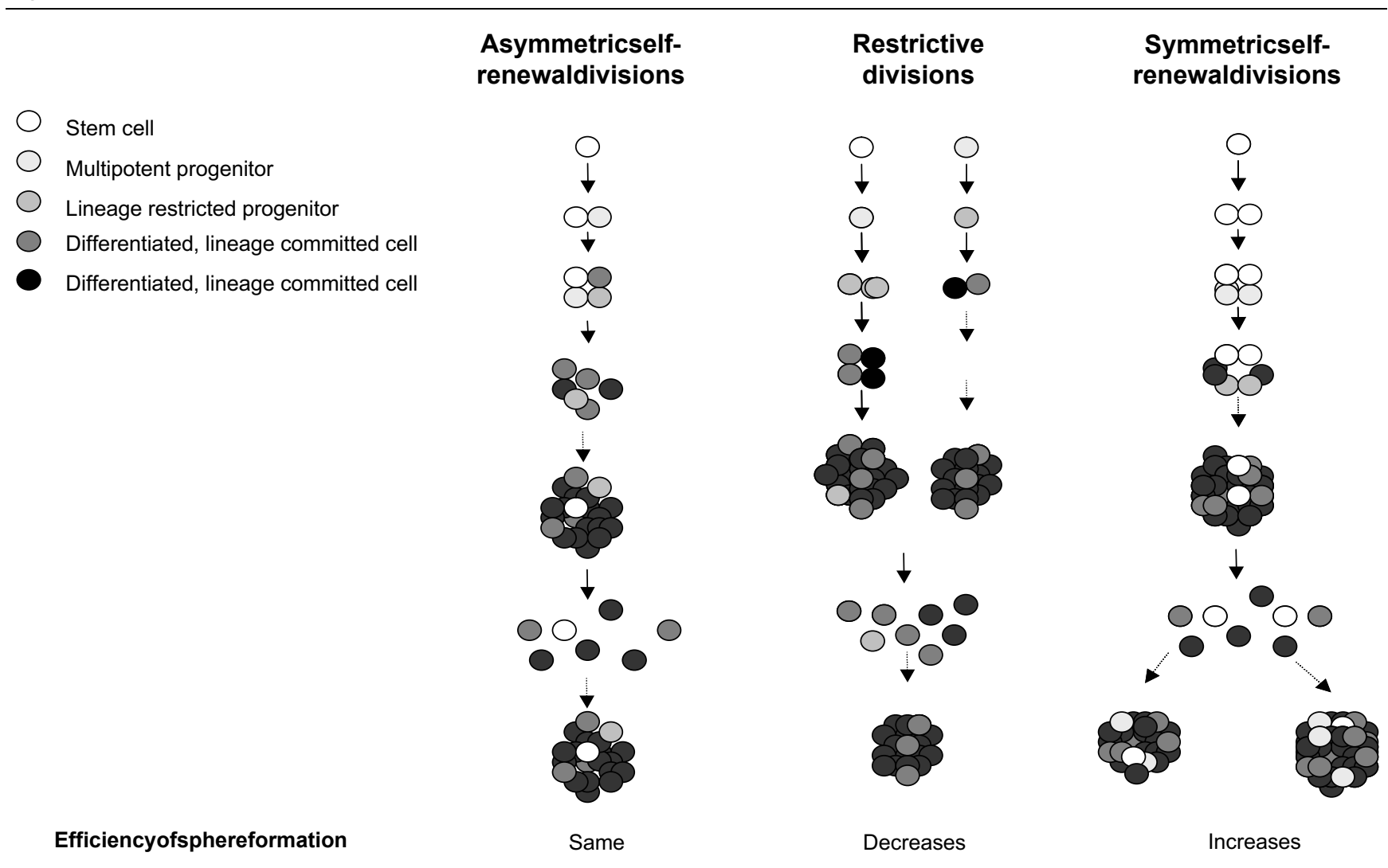

(a)

(b)

(c)

Effect of stem cell self-renewal and restrictive divisions on the efficiency of mammosphere formation. (a) Asymmetric stem cell self-renewal divisions result in constant mammosphere numbers in serial passages. (b) Proliferation of stem cells or progenitor cells through restrictive divisions results in a decreasing number of spheres in serial passages. (c) Symmetric self-renewal divisions of stem cells result in an increasing number of mammospheres in serial passages.

recently been proposed that stem cells or early progenitor cells constitute the primary targets of transformation [2].

We have recently described the existence of a tumorigenic subset of human breast carcinoma cells, with a definable phenotype, which is responsible for tumor formation as well as for generating the phenotypic heterogeneity found in breast cancers [39]. Tumorigenic cells, like their normal stem cell counterparts, can therefore undergo self-renewal as well as differentiation, albeit aberrant. We have postulated that the tumor stem cell population drives tumorigenesis, recurrence and metastasis [40].

In addition to our findings regarding the role of Notch in self-renewal of mammary stem cells, these findings suggest that carcinogenesis may involve the deregulation of processes involved in normal stem cell self-renewal. Elucidation of key elements of these pathways may identify new targets for cancer therapy [32].

\section{Competing interests}

MW has financial holdings in Oncomed, which has applied for a patent on cancer stem cell technologies.

\section{Acknowledgements}

Thanks are due to Dr Thomas Giordano for tissue procurement, the University of Michigan Cancer Center Flow Cytometry core, Dr Michael Clarke for technical advice and Dr Sean Morrison for providing the recombinant Delta 1-Fc recombinant ligand and for critical review of the paper. This work was supported by $\mathrm{NIH}$ grants $\mathrm{CA} 66233$ and CA101860, and in part by the University of Michigan Cancer Center NIH Support Grant 5 P 30 CA46592.

\section{References}

1. Weissman IL: Stem cells: units of development, units of regeneration, and units in evolution. Cell 2000, 100:157-168.

2. Reya T, Morrison SJ, Clarke MF, Weissman IL: Stem cells, cancer, and cancer stem cells. Nature 2001, 414:105-111.

3. Smith GH, Chepko G: Mammary epithelial stem cells. Microsc Res Technique 2001, 52:190-203.

4. Dontu G, Abdallah W, Foley J, Jackson K, Clarke M, Kawamura M, Wicha M: In vitro propagation and transcriptional profiling of 
human mammary stem/progenitor cells. Genes Dev 2003, 17:1253-1270.

5. Reynolds BA, Weiss S: Clonal and population analyses demonstrate that an EGF-responsive mammalian embryonic CNS precursor is a stem cell. Dev Biol 1996, 175:1-13.

6. Miele L, Osborne B: Arbiter of differentiation and death: Notch signaling meets apoptosis. J Cell Physio/ 1999, 181:393-409.

7. Artavanis-Tsakonas S, Rand MD, Lake RJ: Notch signaling: cell fate control and signal integration in development. Science 1999, 284:770-776

8. Qi H, Rand MD, Wu X, Sestan N, Wang W, Rakic P, Xu T, Artavanis-Tsakonas $S$ : Processing of the notch ligand delta by the metalloprotease Kuzbanian. Science 1999, 283:91-94.

9. Li L, Milner LA, Deng Y, Iwata M, Banta A, Graf L, Marcovina S, Friedman C, Trask BJ, Hood L, et al:: The human homolog of rat Jagged1 expressed by marrow stroma inhibits differentiation of 32D cells through interaction with Notch1. Immunity 1998, 8:43-55.

10. Six E, Ndiaye D, Laabi Y, Brou C, Gupta-Rossi N, Istrael A, Logeat $F:$ The Notch ligand Delta1 is sequentially cleaved by an ADAM protease and gamma-secretase. Proc Natl Acad Sci USA 2003, 100:7638-7643.

11. Kadesch $\mathrm{T}$ : Notch signaling: a dance of proteins changing partners. Exp Cell Res 2000, 260:1-8.

12. Smith G, Gallahan D, Diella F, Jhappan C, Merlino G, Callahan R: Constitutive expression of a truncated INT3 gene in mouse mammary epithelium impairs differentiation and functional development. Cell Growth Diff 1995, 6:563-577.

13. Uyttendaele $\mathrm{H}$, Soriano JV, Montesano R, Kitajewski J: Notch4 and Wnt-1 proteins function to regulate branching morphogenesis of mammary epithelial cells in an opposing fashion. Dev Biol 1998, 196:204-217.

14. Soriano JV, Uyttendaele H, Kitajewski J, Montesano R: Expression of an activated Notch4(int-3) oncoprotein disrupts morphogenesis and induces an invasive phenotype in mammary epithelial cells in vitro. Int J Cancer 2000, 86:652-659.

15. Diévart A, Beaulieu N, Jolicoeur P: Involvement of Notch1 in the development of mouse mammary tumors. Oncogene 1999, 18:5973-5981.

16. Brennan $\mathrm{K}$, Brown AMC: Is there a role for Notch signalling in human breast cancer? Breast Cancer Res 2003, 5:69-75.

17. Stingl J, Eaves CJ, Kuusk U, Emerman JT: Phenotypic and functional characterization in vitro of a multipotent epithelial cell present in the normal adult human breast. Differentiation 1998, 63:201-213.

18. Weaver VM, Bissell MJ: Functional culture models to study mechanisms governing apoptosis in normal and malignant mammary epithelial cells. J Mammary Gland Biol Neoplasia 1999, 4:193-201.

19. Shimazaki $T$, Shingo $T$, Weiss $S$ : The ciliary neurotrophic factor/ leukemia inhibitory factor/gp130 receptor complex operates in the maintenance of mammalian forebrain neural stem cells. $J$ Neurosci 2001, 21:7642-7653.

20. Morrison SJ, Perez SE, Qiao Z, Verdi JM, Hicks C, Weinmaster G, Anderson DJ: Transient Notch activation initiates an irreversible switch from neurogenesis to gliogenesis by neural crest stem cells. Cell 2000, 101:488-510.

21. Mumm JS, Schroeter EH, Saxena MT, Griesemer A, Tian X, Pan DJ, Ray WJ, Kopan R: A ligand-induced extracellular cleavage regulates gamma-secretase-like proteolytic activation of Notch1. Mol Cell 2000, 5:197-206.

22. Jarriault S, Brou C, Logeat F, Schroeter EH, Kopan R, Israel A: Signaling downstream of activated mammalian Notch. Nature 1995, 377:355-358.

23. Rudland PS, Barraclough R, Fernig DG, Smith JA: Mammary stem cells in normal development and cancer. In Stem Cells Edited by: Potten CS. New York: Academic Press; 1997:147-232.

24. Nofziger D, Miyamoto A, Lyons KM, Weinmaster G: Notch signaling imposes two distinct blocks in the differentiation of $\mathrm{C2C} 12$ myoblasts. Development 1999, 126:1689-1702.

25. Nickoloff BJ, Qin J-Z, Chaturvedi V, Benning MF, Bonish B, Miele $L$ : Jagged-1 mediated activation of notch signaling induces complete maturation of human keratinocytes through NF-kB and PPARg. Cell Death Diff 2002, 9:842-855.

26. Tanigaki K, Nogaki F, Takahashi J, Tashiro K, Kurooka H, Honjo T: Notch1 and Notch3 instructively restrict bFGF-responsive mulipotent neural progenitor cells to an astroglial fate. Neuron 2001, 29:45-55.

27. Gudjonsson T, Villadsen R, Nielsen HL, Rønnov-Jessen L, Bissel MJ, Petersen OW: Isolation, immortalization, and characterization of a human breast epithelial cell line with stem cell properties. Genes Dev 2002, 16:693-706.

28. Weijzen S, Rizzo P, Braid M, Vaishnav R, Jonkheer S, Zlobin A, Osborne B, Gottipati S, Aster J, Hahn W, et al:: Activation of Notch-1 signaling maintains the neoplastic phenotype in human Ras-transformed cells. Nat Med 2002, 8:979-986.

29. Morrison SJ, Shah NM, Anderson DJ: Regulatory mechanisms in stem cell biology. Cell 1997, 88:287-298.

30. Weissman IL, Anderson DJ, Gage F: Stem and progenitor cells: origins, phenotypes, lineage commitments, and transdifferentiations. Annu Rev Cell Dev Biol 2001, 17:387-403.

31. Krebs L, Xue Y, Norton C, Shutter J, Maguire M, Sundberg J, Gallahan D, Closson V, Kitajewski J, Callahan R, et al.: Notch signaling is essential for vascular morphogenesis in mice. Genes Dev 2000, 14:1343-1352.

32. Siziopikou K, Miao H, Rizzo P, Song L, Selvaggi S, Bashir A, Koerner F, Qin J, Nickoloff BJ, Miele L: Notch signaling is a therapeutic target in breast cancer [abstract 5575]. In: Proceedings of the 94th Annual Meeting of the AACR: 2003 5-9 April; Toronto Philadelphia, PA: AACR; 2003:1277-1278.

33. Imantani A, Callahan R: Identification of a novel NOTCH4/INT-3 RNA species encoding an activated gene product in certain human tumor cell lines. Oncogene 2000, 19:223-231.

34. Fitzgerald $\mathrm{K}$, Harrington $\mathrm{A}$, Leder $\mathrm{P}$ : Ras pathway signals are reqiured for notch-mediated oncogenesis. Oncogene 2000, 19:4191-4198.

35. Rangarajan A, Syal R, Selvarajah S, Chakrabarti O, Sarin A, Krishna S: Activated Notch1 signaling cooperates with papillomavirus oncogenes in transformation and generates resistance to apoptosis on matrix withdrawal through PKB/Akt Virology 2001, 286:23-30.

36. Zagouras $\mathrm{P}$, Stifani $\mathrm{S}$, Blaumueller $\mathrm{CM}$, Carcangiu ML, ArtavanisTsakonas S: Alterations in Notch signaling in neoplastic lesions of the human cervix. Proc Natl Acad Sci USA 1995, 92:6414-6418.

37. Radtke F, Raj K: The role of Notch in tumorigenesis: oncogene or tumour suppressor. Nat Rev Cancer 2003, 3:756-767.

38. Uyttendaele H, Marazzi G, Wu G, Yan Q, Sassoon D, Kitajewski J: Notch4/int-3, a mammary proto-oncogene, is an endothelial cell-specific mammalian Notch gene. Development 1996 122:2251-2259.

39. Al-Hajj M, Wicha M, Benito-Hernandez A, Morrison S, Clarke M: Prospective identification of tumorigenic breast cancer cells. Proc Natl Acad Sci USA 2003, 100:3983-3988.

40. Dontu G, Al-Hajj M, Abdallah W, Clarke M, Wicha M: Stem cells in normal breast development and breast cancer. Cell Proliferation 2003, 36:59-72. 